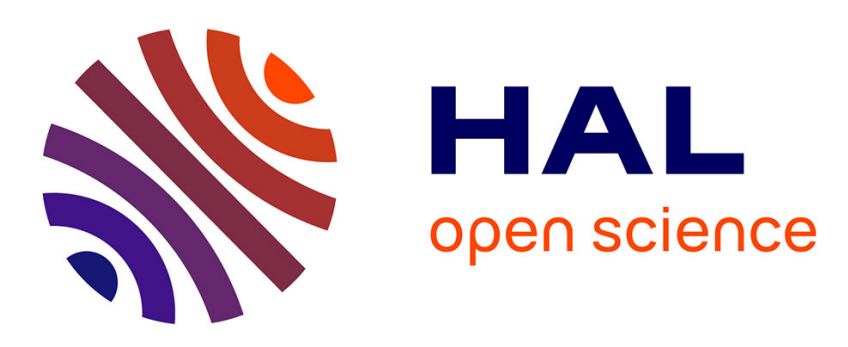

\title{
To what extent does Tagged-MRI technique allow to infer tongue muscles' activation pattern? A modelling study
}

Stéphanie Isabelle Buchaillard, Pascal Perrier, Yohan Payan

\section{To cite this version:}

Stéphanie Isabelle Buchaillard, Pascal Perrier, Yohan Payan. To what extent does Tagged-MRI technique allow to infer tongue muscles' activation pattern? A modelling study. Interspeech 2008 9th Annual Conference of the International Speech Communication Association, Sep 2008, Brisbane, Australia. pp.2839-2842. hal-00334341

\section{HAL Id: hal-00334341 \\ https://hal.science/hal-00334341}

Submitted on 26 Oct 2008

HAL is a multi-disciplinary open access archive for the deposit and dissemination of scientific research documents, whether they are published or not. The documents may come from teaching and research institutions in France or abroad, or from public or private research centers.
L'archive ouverte pluridisciplinaire HAL, est destinée au dépôt et à la diffusion de documents scientifiques de niveau recherche, publiés ou non, émanant des établissements d'enseignement et de recherche français ou étrangers, des laboratoires publics ou privés. 


\title{
To what extent does Tagged-MRI technique allow to infer tongue muscles' activation pattern? A modelling study
}

\author{
Stéphanie Buchaillard ${ }^{1}$, Pascal Perrier ${ }^{1}$, Yohan Payan ${ }^{2}$ \\ ${ }^{1}$ Department of Speech and Cognition, GIPSA-lab, Grenoble, France \\ ${ }^{2}$ TIMC-GMCAO Laboratory, La Tronche, France \\ stephanie.buchaillardegipsa-lab.fr, pascal.perrieregipsa-lab.fr, yohan.payan@imag.fr
}

\begin{abstract}
'Tagged MRI' techniques have been used during the past years to predict which tongue muscles are activated during the production of vowels and for non-speech gestures. Using this technique, tongue muscle activation inferences are based on the hypothesis that a significant distortion of the anatomical region of a tongue muscle is evidence of voluntary muscle activation. In this paper, we propose to use a 3D finite-element model of the oral cavity to study the relation between the strain levels observed in the tongue body in relation to the tongue muscles activated by the central nervous system or through reflex loops. Results showed in most cases a good correlation between the area of the tongue that underwent high strains and the location of the muscle activated when studying single muscle activation, but a limited and even no correlation for movements involving combined muscle activation. A direct reading of Tagged MRI images would not allow inferring major tongue muscles activated in theses cases.
\end{abstract}

Index Terms: Tagged MRI, speech production, biomechanical modelling

\section{Introduction}

Determining experimentally if a tongue or mouth floor muscle is activated during speech or non speech gestures, as well as its level of activation, is not a trivial task. Indeed, a direct exploitation of muscle electrical activity (electromyographic (EMG) data) does not always allow inferring muscles activated, since the fibres of different lingual muscles are often intertwined and the number and distribution of motor units are not well-known. In addition, EMG signals consist of the superposition of descending and afferent signals due to neuromuscular spindles or any other type of proprioceptive feedback: EMG signals are insufficient to distinguish muscles actively recruited from those activated through reflex loops. They do not make it possible to understand how the central nervous system controls the tongue. Therefore, researchers have sought alternative methods to infer recruited muscles, in particular Tagged MRI [1,2] and Tagged cine-MRI images [3, 4]. The principal stretches in the tongue body and their corresponding directions are determined by measuring local tongue deformation. The directions computed are then compared with the muscular fibre orientation of the different lingual tissues in the associated area, in order to infer active muscles. However, the validity of such an approach has not been demonstrated yet.

To evaluate the relationship between the muscles recruited and the strain levels resulting from the muscle activation, a 3D finite-element (FE) model of the oral cavity was used [5]. 11 groups of tongue and mouth floor muscles are represented within the FE structure by specific subsets of elements. Tongue tissues are modelled as a hyperelastic material according to a stress/strain constitutive law adapted from indentation measurements carried out on a fresh cadaver tongue [6,7] and coherent with the in-vivo measurements of [8]. The soft and bony structures surrounding the tongue, such as the jaw, the palate, the pharyngeal and laryngeal walls as well as the hyoid bone, are also modelled. The muscle activations are produced by applying forces acting directly on the FEM structure nodes, along given edges representing muscular fibres. These forces are controlled by an external generator based on a functional model of muscle force generation mechanisms, the $\lambda$ version of equilibrium point hypothesis $(\mathrm{EPH})$ [9]. Every muscle is controlled using a single stretch reflex threshold (the so-called $\lambda$ parameter). For an isometric contraction, the activation is zero when the threshold is greater than the muscle length, and increases when the difference between the muscle length and the threshold increases. The Lagrange equations of motion for the mesh nodes are solved using the Ansys $\AA$ finite element package. The model used can be considered as being realistic in regard to the geometric and physical aspects, as well as its motor control scheme.

In Section 2, the correlation between strains computed following the activation of single muscles and the location of these muscles is introduced. In Section 3, more complex movements are studied, in particular the strain produced during the production of vowel /i/. Conclusions are presented in Section 4.

\section{Analysis of single tongue and mouth floor muscle activation}

The strain levels were studied when activating separately the different tongue and mouth floor muscles alone. In order to illustrate our findings, the results obtained for two muscles only (Fig. 1), the posterior part of the genioglossus (GGp) and the mylohyoid (MH), are presented below (results at the end of a $400 \mathrm{~ms}$ simulation). The activation threshold ( $\lambda$ parameter of the EPH model) for the only activated muscle is fixed to $75 \%$ of the muscle length at rest, and twice higher than the muscle length at rest for non-activated muscles (insuring zero muscular forces for these muscles).

\subsection{Single activation of posterior genioglossus muscle}

The genioglossus muscle, the major tongue muscle, is inserted on the superior mental spine and radiates in three groups of fibres: anterior $(\mathrm{GGa})$, to the apex, middle $(\mathrm{GGm})$ to the deep dorsal mucosa, and posterior horizontal (GGp, Fig. 1(a)). Only the posterior part, whose fibres are nearly horizontal, was acti- 


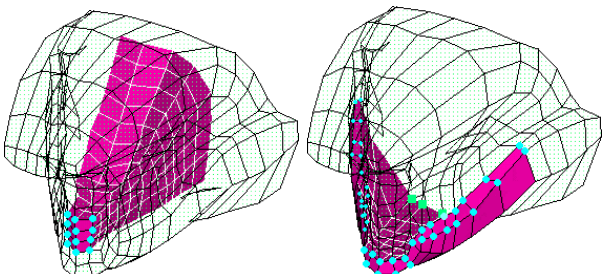

(a) GGp muscle

Figure 1: Location of the GGp and MH muscle (coloured elements) in the tongue mesh structure (transparent) [anterior oblique view]. Squares and dots represent bony insertions.

vated.

The results given Fig. 2 show the first, second and third principal von Mises strain (principal strains, calculated from the strain tensors' eigenvalues, are the values usually exploited for tagged-MRI analysis) as well as the von Mises strain in the midsagittal plane. The von Mises strain map (Fig. 2(d)) shows that main deformations are observed in the area of the tongue corresponding to the GGp location. However, non negligible strain levels are observed in the GGm area (middle part of the tongue above the GGp) as well as in the inferior longitudinalis (lower part of the tongue tip; IL, muscle lateral to the genioglossus, running from the hyoid bone to the tongue tip) area. Similar observations can be made for the first principal strain (Fig. 2(a)). As for the second principal strain map (Fig. 2(b)), higher strains are observed at the intersection of the GGp and the transversalis (Trans, below the dorsum, and radiating from the apex to the rear part of the tongue). High strain levels are also observed in the midsagittal section of the $\mathrm{MH}$. The third principal strain map (Fig. 2(c)) shows a more homogenous repartition of the deformation. In the case of the GGp, only the von Mises strain map and the first principal strain seem to bring reliable information to infer the activated muscle.

\subsection{Single activation of mylohyoid muscle}

The mylohyoid muscle (Fig. 1(b)) is the major muscle of the mouth floor: it extends from the mandible laterally to the hyoid bone medially and supports the anterior floor of the mouth. Its fibres run from the mandibular insertions to the midsagittal plane.

The same results as for GGp are presented Fig. 3. The von Mises strain (Fig. 3(d)) is relatively high in the MH area. However, similar strain levels are observed in the GGp area, of the same order of magnitude as for the single GGp activation. The first principal strain map (Fig. 3(a)) leads to similar observations. Concerning the second principal strain, values are high in the $\mathrm{MH}$ area, and non negligible in the GGp area (similar order of magnitude than previously). For the MH activation alone, precautions need to be taken when analyzing the data: though high level of strains are observed in the GGp area whatever kind of representation is used, this muscle was not actively contracted during the simulation.

\section{Analysis of combined activation of tongue and mouth floor muscles}

The muscular commands necessary to produce French oral vowels were established based on numerous simulations, and

literature data such as EMG data and cineradiographic data. For every simulation, the 3D vocal tract shape was extracted, the area function computed and the corresponding vowel synthetized [10]. Optimal vowels were selected on the basis of the vowels formants.

The results presented in this section were obtained for vowel /i/. The muscles activated and a rough estimation of the associated force levels once the tongue was stabilized are indicated Table 1 (the estimated level of force is computed as the algebraic sum of the force applied one each individual fibre; it is then larger than the actual level of force, since fibres are in general not parallel with each others). Only the GGp, the styloglossus (Sty, muscle whose major bundles of fibres run from the apical area to styloid process, backward and upward), the geniohyoid (GH, flat quadrilateral muscle of the mouth floor) and the MH were voluntarily activated. The GGa and the transversalis were activated through reflex loop mechanisms : these muscles generated forces though their activation thresholds were above their length at rest in order to compensate for their elongation.

Table 1: Final force levels (in Newtons, see text for details) observed for tongue and mouth floor muscles for vowel /i/.

\begin{tabular}{cccccc} 
GGp & GGa & Sty & Trans & GH & MH \\
\hline \hline 23.07 & 1.21 & 12.86 & 0.62 & 3.53 & 13.23
\end{tabular}

The strain maps Fig. 4 reveal that inferring muscle activations from strain levels becomes very difficult as soon as combined muscle activation are brought into play. No serious inference can be made from these different representations. High levels of deformations in the apical area visible on the four maps illustrate the styloglossus activation. Since only a minor part of this muscle is present in the midsagittal plane, its activation can not be visualized in any other part of the map. The GGp activation, though extremely high, is only slightly visible in the midsagittal plane on the first principal strain map (Fig. 4(a)), and in the transversalis area in the second (Fig. 4(b)) and third (Fig. 4(c)) principal strain maps. However, the data are difficult to analyze.

The von Mises strain maps in a coronal plane were also studied, that showed significant strain levels in the GGp area. However, inferring muscle activations from strains is not always effective: in Tagged MRI analysis, compressions observed are supposed to be the result of muscle contractions [4] and can only take into consideration muscles that are directly activated, not muscle activations due to surrounding muscle contractions. For vowel /i/, the von Mises strain map reading in the coronal plane for vowel /i/ could be interpreted as an absence of activation for the transversalis. However, this muscle is indirectly activated (Table 1). During the production of vowel /i/, it limits the deformation of the tongue body in the transverse direction to allow other muscles to be more efficient in the sagittal plane, but does not to generate directly some deformation. It is a major role, but since it is intended to prevent deformation, it cannot be detected by the Tagged MRI technique.

\section{Conclusions}

In conclusion, the Tagged MRI technique can in some cases be used to infer the most active muscle, in particular muscles like the styloglossus, which fibres course and location can be clearly distinguish from the other muscles. However, deep in the tongue body, where numerous tongue fibres are intertwined, the analysis becomes difficult. Due to strain propagation to soft 


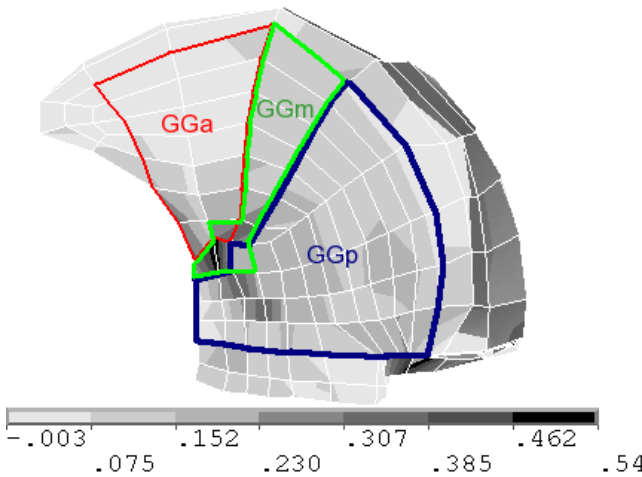

(a) first principal strain

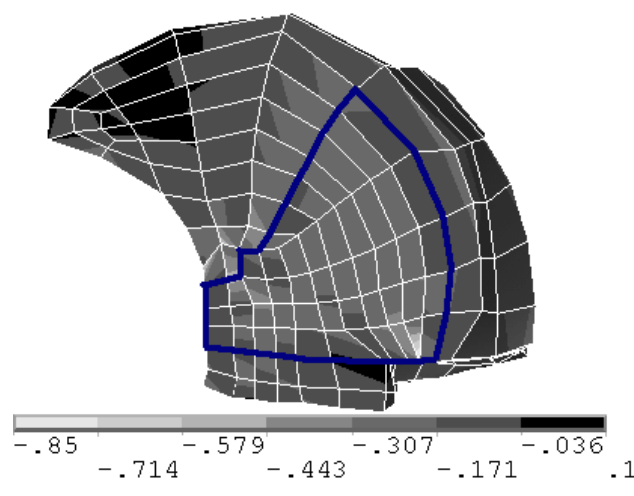

(c) third principal strain

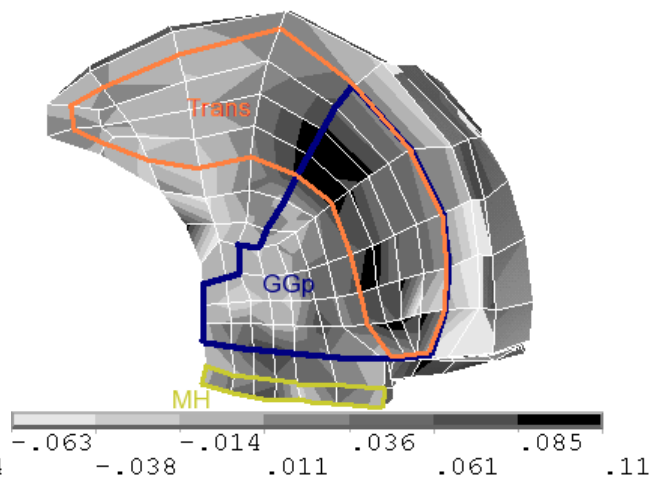

(b) second principal strain

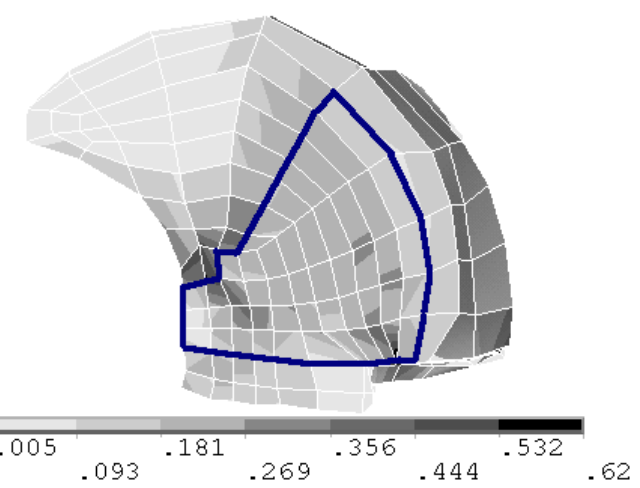

(d) von Mises equivalent strain

Figure 2: Maps of the elastic strain $\left(1^{\text {st }}, 2^{\text {nd }}\right.$ and $3^{\text {rd }}$ principal strains, and equivalent strain) in the midsagittal plane for the activation of the GGp alone [midsagittal cut]. The area outlines of the main tongue muscles are indicated with coloured lines (GGp on every figure).

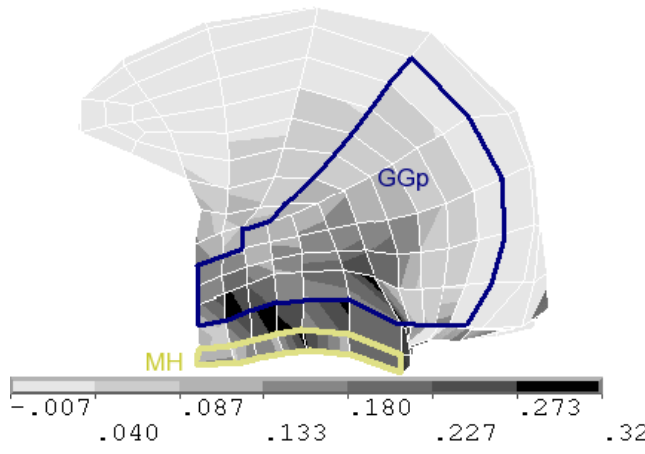

(a) first principal strain

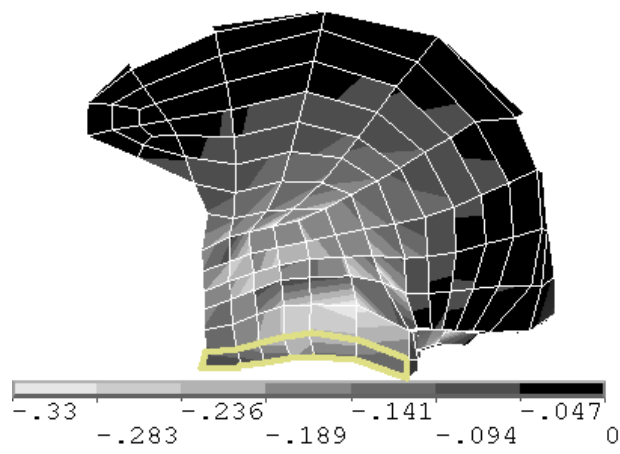

(c) third principal strain

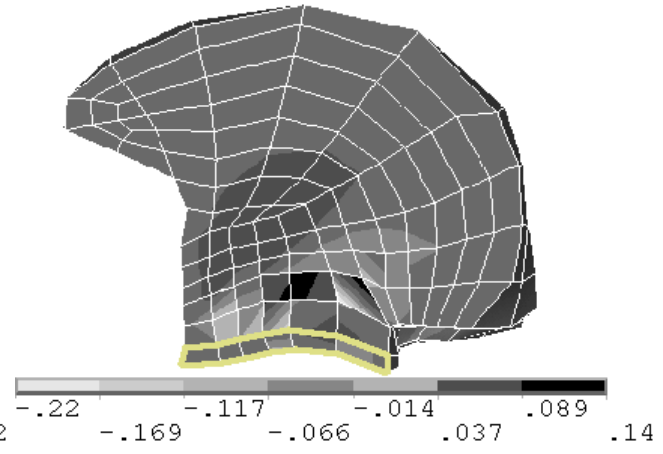

(b) second principal strain

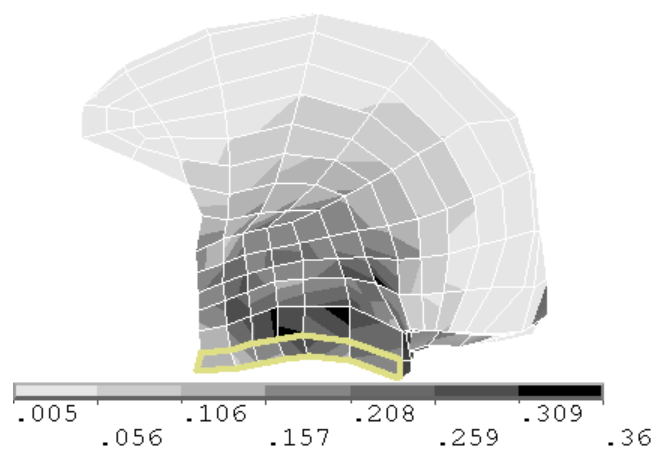

(d) von Mises equivalent strain

Figure 3: Maps of the elastic strain $\left(1^{\text {st }}, 2^{\text {nd }}\right.$ and $3^{\text {rd }}$ principal strains, and equivalent strain) for the activation of the MH alone [midsagittal cut]. The area outlines of the MH and GGp muscles are indicated with coloured lines on Fig. a (MH on every figure). 


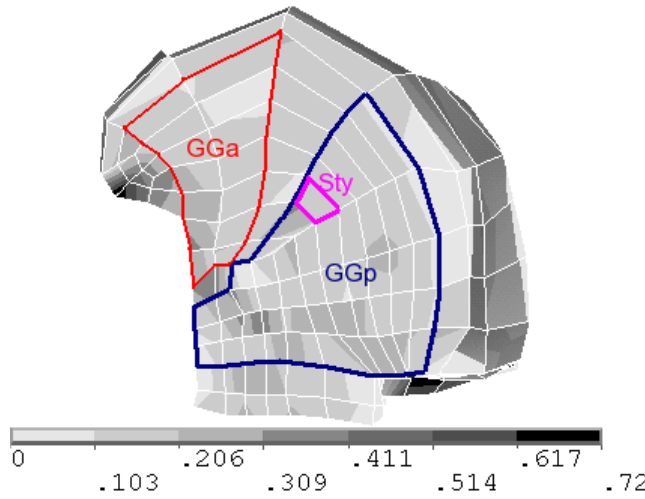

(a) first principal strain

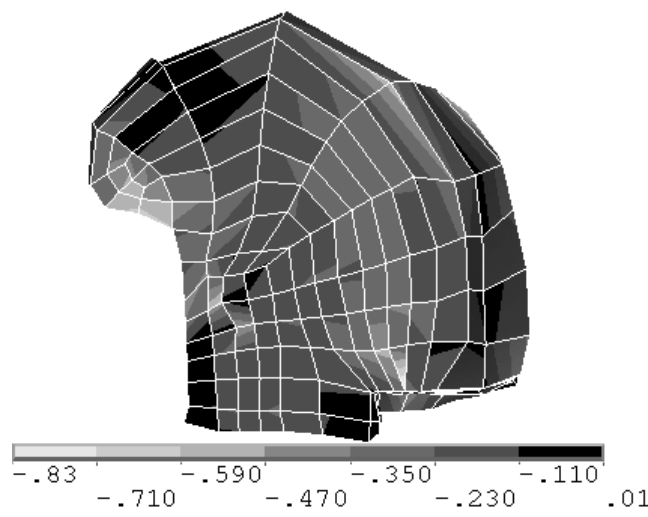

(c) third principal strain

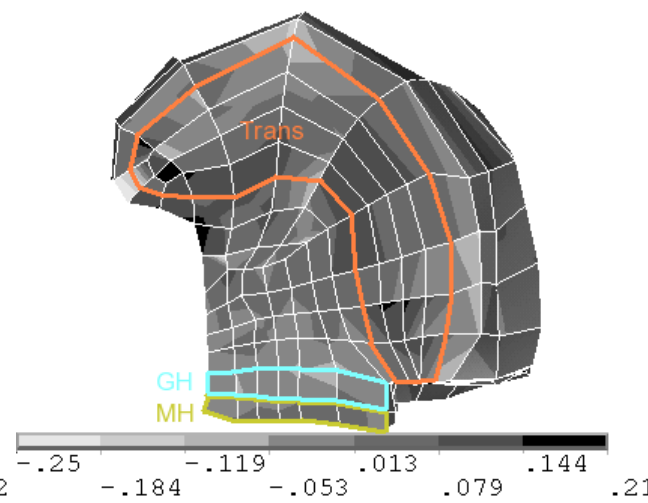

(b) second principal strain

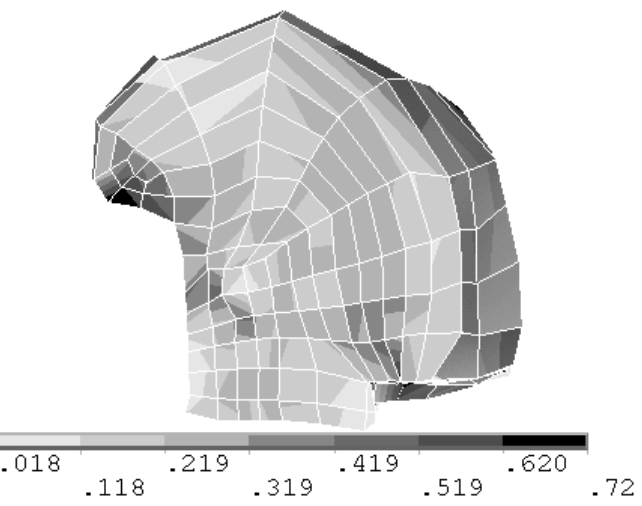

(d) von Mises equivalent strain

Figure 4: Maps of the elastic strain $\left(1^{\text {st }}, 2^{\text {nd }}\right.$ and $3^{\text {rd }}$ principal strains, and equivalent strain) for the production of vowel /i/ [midsagittal cut]. The area outlines of the activated muscles are indicated with coloured lines.

tissues in neighbour areas of the activated region and due to reflex muscle activation, Tagged MRI method does not seem to be reliable to infer the central patterns of activation. Some good knowledge of the independent activation of the different muscle is necessary to interpret the maps. Furthermore, the representations that seem to bring the most interesting information were firstly the von Mises total strain and, secondly, the first principal strain. In addition, looking only at strain maps to detect muscle activations does not offer a comprehensive view of the role of the muscles, since some muscles can be used to keep parts of the tongue constant and to counteract the secondary effect of other muscle activations.

\section{Acknowledgements}

This project was supported in part by the Emergence Program of the Région Rhône-Alpes and by the P2R Program funded by the CNRS and the French Foreign Office (POPAART Project).

\section{References}

[1] K. M. Niitsu, S. Niimi, and Y. Itai, "Tongue movement during phonation: A rapid quantitative visualization using tagging snapshot MRI imaging," Ann. Bull. RILP, vol. 26, pp. 149-156, 1992.

[2] V. J. Napadow, Q. Chen, V. Wedeen, and R. J. Gilbert, "Intramural mechanics of the human tongue in association with physiological deformations," Journal of Biomechanics, vol. 32, pp. 1-12, 1999.

[3] M. Stone, E. P. Davis, A. S. Douglas, M. NessAiver, R. Gullapalli, and A. Levine, William S. andLundberg, "Modeling the motion of the internal tongue from tagged cine-MRI images," J. Acoust. Soc. Am., vol. 109, no. 6, pp. 2974-2982, 2001.

[4] V. Parthasarathy, J. L. Prince, M. Stone, E. Z. Murano, and M. NessAiver, "Measuring tongue motion from tagged cine-MRI using harmonic phase (HARP) processing," J. Acoust. Soc. Am., vol. 121, no. 1, pp. 491-504, 2007.

[5] S. Buchaillard, M. Brix, P. Perrier, and Y. Payan, "Simulations of the consequences of tongue surgery on tongue mobility: Implications for speech production in post-surgery conditions," Int. J. Med. Robot. Comp., vol. 3, no. 3, pp. 252-261, 2007.

[6] J.-M. Gérard, J. Ohayon, V. Luboz, P. Perrier, and Y. Payan, "Non-linear elastic properties of the lingual and facial tissues assessed by indentation technique application to the biomechanics of speech production," Med. Eng. Phys., vol. 27, no. 10, pp. 884 892, 2005.

[7] S. Buchaillard, P. Perrier, and Y. Payan, "A 3D biomechanical vocal tract model to study speech production control: How to take into account the gravity?" in Proceedings of the 7th International Seminar on Speech Production, Ubatuba, Brazil, 2006, pp. $403-$ 410.

[8] P. Schiavone, T. Boudou, J. Ohayon, and Y. Payan, "In-vivo measurement of the human soft tissues constitutive laws. applications to computer aided surgery," Comput. Methods. Biomech. Biomed. Engin., no. Supplement 1, pp. 185-186, 2007.

[9] A. G. Feldman, "Once more on the equilibrium-point hypothesis ( $\lambda$ model) for motor control," J. Mot. Behav., vol. 18, no. 1, pp. 17-54, 1986.

[10] S. Buchaillard, "Activations musculaires et mouvements linguaux : modélisation en parole naturelle et pathologique," $\mathrm{Ph} . \mathrm{D}$. dissertation, Université Joseph Fourier, Grenoble, France, dec 2007. 